\title{
Riesgos psicológicos en adolescentes en situación de pobreza de un pueblo de la selva peruana
}

\section{Psychological risks in adolescents in povertyin a Peruvian jungle town}

\author{
Yndolina Vega Montoya ${ }^{1}$ y Julio Cjuno ${ }^{2 a}$
}

Universidad Católica Los Ángeles de Chimbote, EP Psicología, Pucallpa, Perú ${ }^{1}$ Universidad Católica Los Ángeles de Chimbote, Instituto de Investigación, Chimbote, Perú ${ }^{2}$

ORCID: https://orcid.org/0000-0001-7764-35391

iD ORCID: https://orcid.org/ 0000-0001-6732-0381

Recibido: 16 de julio de 2020

Aceptado: 12 de octubre de 2020

\begin{abstract}
Resumen
El objetivo del presente trabajo es determinar las relaciones de las variables de riesgos psicológicos: satisfacción vital, autoestima, motivación de logro y expectativas de futuro de los adolescentes de un pueblo de la selva peruana que viven en pobreza. La metodología se basa en un estudio transversal, donde se dedujeron frecuencias relativas y absolutas, del mismo modo medidas de tendencia central y de dispersión, así también se analizó las relaciones de las variables a través del Chi cuadrado de Pearson con una confiabilidad del $95 \%$. Los adolescentes involucrados en el estudio tuvieron una edad media de 14 años, la mayoría 69 (53.5\%) fueron de sexo masculino y $37(28.7 \%)$ sin escolaridad. Así también, 102 (79.1\%) reportaron una satisfacción vital baja, $68(52.7 \%)$ autoestima baja, 90 (69.8\%) motivación de logro bajo y 86 (66.7) expectativas del futuro bajo. Se encontró relación significativa entre las variables sexo $(\mathrm{p}=, 011)$ y satisfacción vital $(\mathrm{p}=, 054)$ con expectativas del futuro. Así también, expectativas del futuro se relacionó con la satisfacción vital $(\mathrm{p}=, 054)$ y la autoestima se relacionó con la motivación de logro $(\mathrm{p}=, 004)$. Todas las variables de riesgos psicológicos reportan alta prevalencia del nivel bajo, el sexo y no tener escolaridad influyen 1de forma significativa, reforzado con la condición de pobreza en el que viven. Se requiere promover la atención de la salud psicológica de esta población a través de implementación del servicio de salud mental comunitaria en la selva peruana.
\end{abstract}

Palabras clave: Autoestima, expectativas del futuro, motivación de logro, satisfacción vital, riesgos psicológicos. 


\begin{abstract}
The objective of this work is to determine the relationships of the psychological risk variables: life satisfaction, self-esteem, achievement motivation and future expectations of adolescents from a Peruvian jungle town who live in poverty. The methodology is based on a cross-sectional study, where relative and absolute frequencies were deduced, in the same way measures of central tendency and dispersion, thus the relationships of the variables were also analyzed through Pearson's Chi square with a reliability of $95 \%$. The adolescents involved in the study had a mean age of 14 years, the majority 69 (53.5\%) were male and $37(28.7 \%)$ without schooling. Also, 102 (79.1\%) reported low life satisfaction, 68 (52.7\%) low self-esteem, 90 (69.8\%) low achievement motivation, and 86 (66.7) low future expectations. A significant relationship was found between the variables sex $(p=.011)$ and life satisfaction $(p=.054)$ with future expectations. Likewise, future expectations were related to life satisfaction $(p=.054)$ and self-esteem was related to achievement motivation $(\mathrm{p}=.004)$. In conclusion, All the psychological risk variables report a high prevalence of the low level, sex and not having schooling have a significant influence, reinforced by the condition of poverty in which they live. It is necessary to promote the psychological health care of this population through the implementation of the community mental health service in the Peruvian jungle.
\end{abstract}

Keywords: Self-esteem, future expectations, achievement motivation, life satisfaction, psychological risks

\title{
Introducción
}

La generalidad de indigentes del planeta se ubica en áreas periféricas y con poca preparación académica, dedicados a las actividades agrícolas y son menores de dieciocho años. Estas limitaciones se muestran en cuanto a educación, atención en salud, alumbrado eléctrico y agua saludable los que se mantienen externamente sin alcance de numerosos individuos, por situaciones socioeconómicas, territoriales, características propias y de género (Leary, 2015)

Aquellas limitaciones dificultan ofrecer un servicio sanitario y de salud mental a los que aún están en extrema necesidad; por su ubicación en territorios frágiles y relieves remotos, por lo que la mejora es transitoria de aquellos que han podido emerger de la escasez: el desbalance monetario, desequilibrio alimenticio y los fenómenos meteorológicos fueron barreras para que los gobiernos puedan brindar una adecuada atención de sus necesidades de salud psicológica (Banco Mundial, 2014)

De acuerdo al Instituto Nacional de Estadística e Informática en el 2018, la brecha educativa, mostró que el 48,7 \% de adolescentes pobres y el $24.3 \%$ de los no pobres estudiaron la primaria y otros no estudiaron. En secundaria los pobres y no pobres casi tuvieron los mismos porcentajes, es decir 42,9\% y 41,6\%, el contraste se nota en formación universitaria, en el cual 34,1\% que cuentan con recursos han adquirido educación superior, en 
tanto que los pobres, solo $8,4 \%$, lo cual también se percibe por el lugar donde viven, los pobres de la ciudad consiguieron educarse 8,3 años, y 5,8 años quienes viven en la periferia. Así mismo los no pobres de la ciudad consiguen estudiar 10,7 años y los de rural 6,9 años (INEI, 2018)

Dada la situación descrita anteriormente, la investigación se centró en los riesgos psicológicos de los adolescentes de la selva peruana. Cabañero, Richard, Caballero, Orts, Reig, et. definen la satisfacción vital como la valoración global que el individuo crea de su existencia, comparando, sus beneficios, con lo que buscaba, sus perspectivas (2004). Asimismo, Branden define a la autoestima como la experiencia primordial donde alcanzamos transportar características y practicar requerimientos como la seguridad de nosotros para recapacitar y poder hacer frente a los retos en nuestra existencia, desarrollarnos y alcanzar la felicidad; asumiendo responsabilidades con dignidad, resaltando valores y aprovechar el producto esmerado (Branden, 2011), otros estudiosos también lo definen como la expectativa de obtención de metas, una de las variables tocadas en el estudio y la motivación de logro, que está determinada por tres elementos vitales: el sentido del sí mismo, los estímulos propios y las opciones observadas (Allan \& Ballesteros, 2015) Sumado a lo que ya se ha explicado, los adolescentes del Asentamiento Humano Las Melinas-Perú, no son ajenos a la realidad descrita en cuanto a las brechas de educación, económicos, salud psicológica o salud mental por la condición y limitado acceso a los servicios básicos. Por tal situación se formuló el presente estudio con el objetivo, determinar las relaciones de los riesgos psicológicos en adolescentes del Asentamiento Humano Las Melinas que viven en pobreza.

\section{Metodología}

\section{Diseño de la investigación}

Este estudio fue transversal, debido a que los resultados se obtuvieron en un solo memento, tal como se manifiestan los elementos de la investigación, en el lugar de los hechos (Gordis, 2014).

\section{Población y contexto}


La presente investigación se realizó con los adolescentes del asentamiento humano Las Melinas, dicho lugar se encuentra en el distrito de Manantay del Departamento de Ucayali, según su ubicación geográfica se sitúa en el cono sur de esta región, con un relieve es plano, su clima es tropical (cálido húmedo y lluvioso) su temperatura promedio entre $36 \mathrm{y}$ $40{ }^{0} \mathrm{C}$, casi todo el año, con dos estaciones notable invierno y verano (Centro Nacional de Epidemiologia, 2018).

Se ha observado que, en el Asentamiento Humano Las Melinas, existen muchas carencias como falta de servicios básicos, servicios de salud mental y centros educativos ocasionando una serie de problemas entre ellos de salud psicológica. Este asentamiento tiene una población de 400 habitantes de todas las generaciones, cuyos ingresos económicos familiares son bajos, siendo sus actividades cotidianas la caza, pesca y agricultura. En este contexto, se contó con una población ( $\mathrm{N}=176)$ adolescentes, de ello, se extrajo una muestra no probabilística de $n=129$ adolescentes en situación de pobreza. La situación de pobreza se determinó en la Selva urbana en base al ingreso familiar mensual no mayor a 180 soles y en zona rural 146 soles (Instituto Nacional de Estadistica e Informatica, 2018).

\section{Variables de la investigación}

Las variables principales fueron la satisfacción vital que fue medido por la Escala de satisfacción vital en sus tres niveles (alto/medio/bajo) Alfa de Cronbach de 0.79 (Diener, Emmons, Larsen, \& Griffin, 1985), autoestima evaluado por la escala abreviada de Rosemberg constituida por cinco niveles (alta/tendencia alta/media/tendencia baja/baja), dicho instrumento tiene una confiabilidad de Alpha de Cronbach 0.78 y se efectuó el análisis de consistencia interna, que para todos los modelos fue bueno $(\mathrm{H}>.80)$ (Vanessa Smith Castro, 2014), asimismo motivación de logro calculado por la escala de motivación de logro en tres niveles (alto/medio/bajo), con alfa de Cronbach de 0.86 , y para las sub-escalas (aplicando la corrección de Spearman son del orden de 0.90; Mas \& Alonso, 1998), finalmente expectativas acerca del futuro examinado por la escala de desesperanza de Beck con sus niveles (alto/medio/bajo), el coeficiente alfa de Crombach es de 0.80 , mientras que el análisis factorial con la técnica de los componentes principales y rotación varimax encuentra 6 factores que explican el $49.32 \%$ de la varianza (Aliaga-Tovar et al., 2014). Las variables secundarias fueron: sexo (femenino y masculino), estado civil de los padres (casado, soltero, conviviente), grado de instrucción de los padres (analfabetos, primaria y secundaria), tipo de familia (monoparentales, nuclear, extensa, reconstituidas). 


\section{Procedimiento}

El proceso de la investigación se dio de la siguiente manera, se realizó las coordinaciones en el Asentamiento Humano Las Melinas, buscando el permiso del presidente del lugar, luego se solicitó la autorización para aplicar los instrumentos de investigación mediante una encuesta, la cual se realizó casa por casa explicándoles a cada participante los objetivos del estudio y el consentimiento informado, que en caso de ser menores de edad fueron firmados por sus padres y además el asentimiento informado por el adolescente. Posterior a ello, se realizó la aplicación del instrumento que fue realizado por encuestadores. Se empleó la técnica de la entrevista, pues el encuestador fue quien registró los resultados en función a la información brindada por el participante.

Los datos fueron digitados por dos trabajadores y posteriormente se cruzaron los datos, en caso de errores de digitación se recorrieron a las encuestas en físico para verificar la respuesta correcta, se realizó dicha actividad hasta lograr tener una base de datos limpia.

\section{Análisis estadístico}

En el análisis univariado se usó la distribución de frecuencias relativas y porcentuales para las variables categóricas, y las medidas de tendencia central y dispersión para las variables numéricas. Por otro lado, en el análisis bivariado, para probar la dependencia o independencia de las variables de caracterización como: edad, sexo, escolaridad) y las variables psicológicas: autoestima, expectativas del futuro, satisfacción vital y motivación del logro, se realizó la prueba estadística de Chi cuadrado de Pearson, siendo significativa con un $\mathrm{p}<0.05$. Se utilizó el programa estadístico SPSS versión 22 y Excel 2016.

\section{Aspectos éticos}

El presente estudio, fue aprobado por el Comité Institucional de Ética en Investigación de la Universidad católica Los Ángeles de Chimbote, asimismo se respetaron todos los principios éticos de investigación en humanos como el principio de justicia, confidencialidad, autonomía.

\section{Resultados}

En el análisis univariado, los adolescentes que participaron en el presente estudio tuvieron una edad media de 14 años, en su mayoría 69 (53.5\%) fueron de sexo masculino, 37 
(28.7\%) fueron analfabetos. Así también, 102 (79.1\%) reportaron una satisfacción vital baja, 68 (52.7\%) autoestima baja, 90 (69.8\%) motivación de logro bajo y 86 (66.7) expectativas del futuro bajo (tabla 1).

\section{Tabla 1}

Características de los adolescentes de la Provincia de Coronel Portillo, Ucayali-Perú

\begin{tabular}{lc} 
Variables & $\mathbf{n ~ ( \% )}$ \\
\hline Edad media (DS) & $14.50 \pm 0,614$ \\
Sexo & \\
Femenino & $60(46.5)$ \\
Masculino & $69(53.5)$ \\
Escolaridad & \\
Secundaria & $10(7.8)$ \\
Primaria & $82(63.6)$ \\
Analfabeto & $37(28.7)$ \\
Satisfacción vital & \\
Alto & $14(10.9)$ \\
Medio & $13(10.1)$ \\
Bajo & $102(79.1)$
\end{tabular}

\section{Autoestima}

Tendencia alta

Media

Tendencia baja

Baja

$68(52.7)$

\section{Motivación de logro}

$\begin{array}{ll}\text { Alto } & 15(11.6) \\ \text { Medio } & 24(18.6) \\ \text { Bajo } & 90(69.8) \\ \text { Expectativas del futuro } & \\ \text { Alto } & 13(10.1) \\ \text { Medio } & 30(23.3) \\ \text { Bajo } & 86(66.7)\end{array}$

En el análisis bivariado, las variables sexo $(\mathrm{p}=, 011)$ y satisfacción vital $(\mathrm{p}=, 054)$ se relacionaron significativamente con expectativas del futuro (tabla 2). 


\section{Tabla 2}

Factores relacionados a expectativas del futuro en adolescentes de la Provincia de Coronel Portillo, Ucayali-Perú

\begin{tabular}{|c|c|c|c|c|}
\hline \multirow[t]{2}{*}{ Variables } & \multicolumn{4}{|c|}{ Expectativas del futuro } \\
\hline & $\begin{array}{l}\text { Bajo } \\
\text { n(\%) }\end{array}$ & $\begin{array}{c}\text { Medio } \\
\text { n(\%) }\end{array}$ & $\begin{array}{l}\text { Alto } \\
\text { n(\%) }\end{array}$ & $P$ Valor \\
\hline \multicolumn{5}{|l|}{ Edad } \\
\hline Menor a 14 años & $49(57.0)$ & $11(36.7)$ & $6(46.2)$ & \multirow{2}{*}{148} \\
\hline Mayor a 15 años & $37(43.0)$ & $19(63.3)$ & $7(53.8)$ & \\
\hline \multicolumn{5}{|l|}{ Sexo } \\
\hline Femenino & $33(38.4)$ & $21(70.0)$ & $6(46.2)$ & \multirow{2}{*}{, 011} \\
\hline Masculino & $53(61.6)$ & $9(30.0)$ & $7(53.8)$ & \\
\hline \multicolumn{5}{|l|}{ Escolaridad } \\
\hline Secundaria & $8(9.3)$ & $2(6.7)$ & $0(0.0)$ & \multirow{3}{*}{,408 } \\
\hline Primaria & $56(65.1)$ & $16(53.3)$ & $10(76.9)$ & \\
\hline Analfabeto & $22(25.6)$ & $12(40.0)$ & $3(23.1)$ & \\
\hline \multicolumn{5}{|l|}{ Satisfacción vital } \\
\hline Alto & $9(10.5)$ & $1(3.3)$ & $4(30.8)$ & \multirow{3}{*}{,054 } \\
\hline Medio & $11(12.8)$ & $2(6.7)$ & $0(0.0)$ & \\
\hline Bajo & $66(76.7)$ & $27(90.0)$ & $9(69.2)$ & \\
\hline \multicolumn{5}{|l|}{ Autoestima } \\
\hline Tendencia alta & $8(9.3)$ & $2(6.7)$ & $1(7.7)$ & \multirow{4}{*}{, 447} \\
\hline Media & $10(11.6)$ & $2(6.7)$ & $0(0.0)$ & \\
\hline Tendencia baja & $22(25.6)$ & $9(30.0)$ & $7(53.8)$ & \\
\hline Baja & $46(53.5)$ & $17(56.7)$ & $5(38.5)$ & \\
\hline
\end{tabular}

Así también, la variable, expectativas del futuro se relacionó significativamente con la satisfacción vital $(\mathrm{p}=, 054)$ (tabla 3$)$.

\section{Tabla 3}

Factores relacionados a la satisfacción vital de los adolescentes de la Provincia de Coronel Portillo, Ucayali - Perú 


\section{Satisfacción vital}

$\begin{array}{cccc}\text { Bajo } & \text { Medio } & \text { Alto } & \text { P valor } \\ \mathrm{n}(\%) & \mathrm{n}(\%) & \mathrm{n}(\%) & \end{array}$

\section{Edad}

Menor a 14 años

$49(48.0) \quad 7(53.8) \quad 10(71.4)$

Mayor a 15 años

$53(52.0)$

$6(46.2)$

$4(28.6)$

, 254

Sexo

Femenino

Masculino

$\begin{array}{rrrr}51(50.0) & 2(15.4) & 7(50.0) & , 060 \\ 51(50.0) & 11(84.6) & 7(50.0) & \end{array}$

Escolaridad

Secundaria

$$
10(9.8)
$$

$0(0.0)$

$0(0.0)$

Primaria

$66(64.7)$

$8(61.5)$

$8(57.1)$

Analfabeto

$26(25.5)$

$5(38.5)$

$6(42.9)$

\section{Autoestima}

Tendencia alta

$$
8(7.8)
$$

2 (15.4)

$1(7.1)$

Media

Tendencia baja

$8(7.8)$

$2(15.4)$

2 (14.3)

29 (28.4)

$3(23.1)$

$6(42.9)$

,668

Baja

57 (55.9)

$6(46.2)$

$5(35.7)$

\section{Motivación de logro}

Alto

$12(11.8)$

$3(23.1)$

$0(0.0)$

Medio

21 (20.6)

$0(0.0)$

$3(21.4)$

,191

Bajo

$69(67.6)$

$10(76.9)$

$11(78.6)$

Expectativas del futuro

$\begin{array}{lrrrr}\text { Alto } & 9(8.8) & 0(0.0) & 4(28.6) & \\ \text { Medio } & 27(26.5) & 2(15.4) & 1(7.1) & \mathbf{0 5 4} \\ \text { Bajo } & 66(64.7) & 11(84.6) & 9(64.3) & \end{array}$

Mientras que, la motivación de logro se relacionó significativamente con la autoestima $(p=, 447)($ tabla 4$)$.

\section{Tabla 4}

Factores relacionados a la autoestima de los adolescentes de la Provincia de Coronel Portillo, Ucayali - Perú 


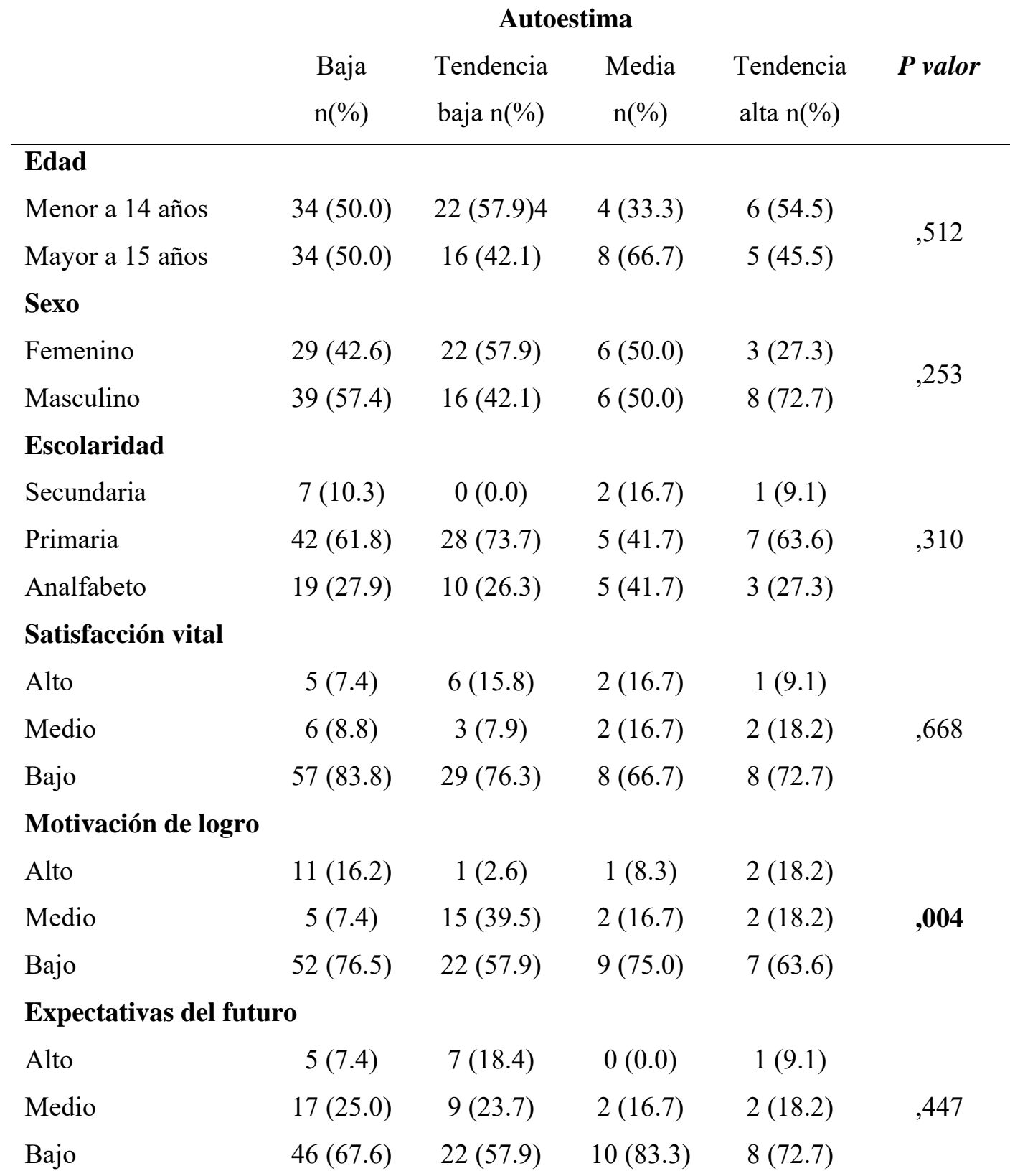

Por último, la autoestima se relacionó significativamente con la motivación de logro $(p=, 004)($ tabla 5$)$.

\section{Tabla 5}

Factores relacionados con la motivación de logro en los adolescentes de la Provincia de Coronel Portillo, Ucayali - Perú 


\section{Motivación de Logro}

Bajo $\mathrm{n}(\%) \quad$ Alto $\mathrm{n}(\%) \quad \boldsymbol{P}$ Valor
$\mathrm{n}(\%)$

\section{Edad}

Menor a 14 años

$47(52.2) \quad 12(50.0) \quad 7(46.7)$

Mayor a 15 años

$43(47.8) \quad 12(50.0) \quad 8(53.3)$

,916

Sexo

Femenino

Masculino

$38(42.2) \quad 15(62.5) \quad 7(46.7)$

Escolaridad

Secundaria

$52(57.8)$

$9(37.5) \quad 8(53.3)$

Primaria

$7(7.8)$

$1(4.2)$

2 (13.3)

Analfabeto

$55(61.1) \quad 16(66.7)$

$11(73.3)$

, 598

\section{Satisfacción vital}

$\begin{array}{lcccc}\text { Alto } & 11(12.2) & 3(12.5) & 0(0.0) & \\ \text { Medio } & 10(11.1) & 0(0.0) & 3(20.0) & , 191 \\ \text { Bajo } & 69(76.7) & 21(7.5) & 12(80.0) & \end{array}$

\section{Expectativas del futuro}

$\begin{array}{lccc}\text { Alto } & 9(10.0) & 4(16.7) & 0(0.0) \\ \text { Medio } & 19(21.1) & 7(29.2) & 4(26.7) \\ \text { Bajo } & 62(68.9) & 13(54.2) & 11(73.3) \\ \text { Autoestima } & & & \\ \text { Tendencia alta } & 7(7.8) & 2(8.3) & 2(13.3) \\ \text { Media } & 9(10.0) & 2(8.3) & 1(6.7) \\ \text { Tendencia baja } & 22(24.4) & 15(62.5) & 1(6.7) \\ \text { Baja } & 52(57.8) & 5(20.8) & 11(73.3)\end{array}$

\section{Discusión}

El presente estudio se propuso determinar la relación entre las variables psicológicas como satisfacción vital, autoestima, motivación de logro y expectativas de futuro con las variables sociodemográficas en adolescentes del asentamiento humano Las Melinas, UcayaliPerú que viven en pobreza. Encontramos que las variables sexo y satisfacción vital se relacionaron significativamente con expectativas de futuro, existió una relación en ambas direcciones entre la variable satisfacción vital y expectativas del futuro. Esto podría darse, debido que los adolescentes de sexo masculino generalmente tienen un desarrollo emocional a 
mayor edad, sumado a eso una baja satisfacción con su vida dentro de la familia, haciéndolo ver pocas oportunidades de logro o éxito en sus planes o metas trazadas, afectando así sus expectativas sobre su futuro (Aktop \& Erman, 2006).

Se encontró una relación significativa entre la motivación de logro y autoestima en doble dirección. El origen puede ser una baja motivación para lograr metas de un adolescente, que se debe a constantes refuerzos experimentados que construyen sentimientos de ser un fracasado o menos que sus iguales, desencadenando así una percepción negativa de su autoestima (Yaffe, 2020). Asimismo, existen estudios similares en adolescentes como el realizado en Colombia, con 821 adolescentes entre 13 y 19 años, en tales resultados presentan una autoestima baja $(76,7 \%)$ y solo el $(1,63 \%)$ presentan nivel esperados de motivación de logro (Andrade Salazar José Alonso \& Gonzáles Portillo Juan, 2017).

Por otro lado, en México un estudio con 1.093 adolescentes que viven en pobreza de edad promedio de 14 años, cuyos resultados indican que el 36\% de los adolescentes tenían una actitud positiva hacia el futuro (Palomar-Lever, Victorio-Estrada, Palomar-Lever, \& Victorio-Estrada, 2017). Como se puede apreciar los niveles bajos de estas variables en otras poblaciones parecidas, evidencian porcentajes similares a los que encontramos. Estos resultados podrían ser debido al proceso de desarrollo del adolescente que lo encuentra a nivel psicológico con diversas dudas e inseguridades, logrando así afectar en la identidad y estima personal, sus expectativas del futuro, por ende, tampoco podría sentir una motivación para lograr metas trazadas ni una satisfacción acerca de su vida (Ahmed et al., 2017).

\section{Limitaciones y fortalezas del estudio}

Una de las limitaciones del presente estudio fue que no se empleó un muestreo probabilístico, pero como nuestra muestra supera el $60 \%$ de la población y se dio en una población de adolescentes hijos de agricultores, cazadores y pescadores en situación de pobreza, su valor no es desestimado. Asimismo, una de nuestras fortalezas es que se trata del primer estudio en una población de difícil acceso y situación de pobreza en la selva peruana. Otra limitación, es que el modelo de análisis no fue epidemiológico por ende es probable que existan más variables con las que no logramos relacionar, que pudieron dar un mayor ajuste al estudio, sin embargo, aquello no le quita el valor en un modelo de análisis estadístico que también es válido.

\section{Conclusiones}

Los adolescentes del distrito de Manantay (Ucayali-Perú) que viven en situación de pobreza reportaron una relación significativa entre sexo y el nivel de satisfacción vital con 
expectativas de futuro; mientras que, la motivación de logro y autoestima también se relacionaron. Se reportaron altas prevalencias negativas en las variables estudiadas. Las autoridades de salud del Perú y tomadores de decisiones pueden hacer uso de nuestros resultados para promover políticas que implementen personal de salud mental y de apoyo psicológico que promueva el bienestar en la autoestima, expectativas del futuro, satisfacción vital y motivación del logro en los adolescentes en situación de pobreza de la selva peruana. Futuros estudios en esta población podrían analizar la causalidad y magnitud de los factores que generan riesgos psicológicos relacionados a la autoestima, satisfacción vital, motivación del logro y expectativas del futuro en adolescentes de la selva peruana.

\section{Referencias}

Ahmed, M. D., Ho, W. K. Y., Van Niekerk, R. L., Morris, T., Elayaraja, M., Lee, K.-C., \& Randles, E. (2017). The self-esteem, goal orientation, and health-related physical fitness of active and inactive adolescent students. Cogent Psychology, 4(1), 1331602. https://doi.org/10.1080/23311908.2017.1331602

Aktop, A., \& Erman, K. A. (2006). Relationship between achievement motivation, trait anxiety and self-esteem. Biology of Sport, 23(2), 127-141. http://31.186.81.235:8080/api/files/view/14020.pdf

Aliaga Tovar, J., Rodríguez de los Ríos, L., Ponce Díaz, C., Frisancho León, A., Enríquez Vereau, J., \& Vereau, J. E. (2014). Escala de desesperanza de Beck (BHS): adaptación y características psicométricas. Revista de Investigación en Psicología, 9(1), 69. https://doi.org/10.15381/rinvp.v9i1.4029

Allan, M., \& Ballesteros, M. (2015). Validación de la Escala de Motivación de Logro Escolar (. Psicothema, 15(2), 333-351. http://www.scielo.org.mx/scielo.php?pid=S166526732015000200009\&script=sci_arttext

Andrade Salazar José Alonso, \& Gonzáles Portillo Juan. (2017). Relación entre riesgo suicida, autoestima, desesperanza y estilos de socialización parental en estudiantes de bachillerato. Universidad Simón Bolívar Colombia, 20(37), 70-88.URL

Branden, N. (2011). El poder de la autoestima (1 edición). Barcelona : Octubre 2011. Recuperado de http://dec.fca.unam.mx/imss/2013/dip_admonestrategica/mod2/EL PODER DE LA AUTOESTIMA.pdf 
Cabañero Martinez, J., Richard Martinez, M., Caballero Garcia, J., Orts Cortes, I., Reig Ferrer, A., \& TosalHerrero, B. (2004). Fiabilidad y Validez de la Escala de Satisfacción con la Vida de Diener en una muestra de mujeres embarazadas y puerperas. Psicothema, 16(3), 448-455. https://dps.ua.es/es/documentos/pdf/1999/fiabilidad-y-validez.pdf

Centro Nacional de Epidemiologia, P. y C. de E. - M. (2018). Número de casos de dengue. Perú 2013 - 2017 y 2018*. Lima. http://www.dge.gob.pe/portal/docs/vigilancia/sala/2018/SE03/dengue.pdf

Diener, Emmons, Larsen, \& Griffin. (1985). Compendio de Instrumentos de Medición IIP. (Castro Smith, Ed.). Costa Rica.

Gordis, L. (2014). Epidemiología (El Servier (ed.); quinta). https://www.elsevier.com/books/epidemiology/gordis/978-1-4557-3733-8

Informe del Banco Mundial: Los datos y las mediciones son fundamentales para poner fin a la pobreza extrema. (2014).

Instituto Nacional de Estadistica e Informatica. (2018). Evolucion de la Pobreza Monetaria 2007 - 2018. INEI. Lima: INEI.

https://www.inei.gob.pe/media/MenuRecursivo/publicaciones_digitales/Est/Lib1646/libr o.pdf

Leary, M. (2015). Pobreza: Panorama general.

Mas, M., \& Alonso, A. (1998). Escala Atribucional de Motivación de Logro. Psicothema, 10(2), 333-351. https://doi.org/10.4067/S0718-07052013000100005

Palomar-Lever, J., Victorio-Estrada, A., Palomar-Lever, J., \& Victorio-Estrada, A. (2017). Expectativas educativas de adolescentes mexicanos en condiciones de pobreza. Revista de psicología (Santiago), 26(1), 54-64. https://doi.org/10.5354/0719-0581.2017.46393

Vanessa Smith Castro. (2014). Compendio de Instrumentos de Medicion IIP,. Costa Rica.

Yaffe, Y. (2020). Does self-esteem mediate the association between parenting styles and imposter feelings among female education students? Personality and Individual Differences, 156. https://doi.org/10.1016/j.paid.2019.109789 